\title{
Accretion onto Black Holes
}

\section{Myeong-Gu Park*}

Kyungpook National University, Daegu 41566, KOREA

E-mail: mapdknu.ac.kr

Most energetic events or objects in the universe are explained by accretion onto black holes. However, the accretion flow onto black holes can assume diverse forms. It can be spherical or disk-like, hot or cold, optically thick or thin, highly luminous or under-luminous, efficient or inefficient in terms of energy production, even for the same physical conditions of gas from which black holes accrete. I will review each form of accretion flow with its physical characteristics. I will especially discuss the mass accretion rate and its dependence on the density, temperature, and the angular momentum of gas being accreted. The mass accretion rate is the most important factor to affect the radiation efficiency and the luminosity, and thereby, the growth history of the black holes and their coevolution with host galaxies. The ubiquity of outflow from hot accretion flow and its implications are also discussed.

35th International Cosmic Ray Conference - ICRC2017

10-20 July, 2017

Bexco, Busan, Korea

${ }^{*}$ Speaker. 


\section{Introduction}

Everybody blames black holes, for almost everything. Any energetic or exotic events or objects are likely to be explained by processes that involve black holes. Especially, the accretion onto black holes is the most preferred way to produce large amount of energies or high energy photons or particles within a limited time or volume. We now have firm evidence that stellar mass black holes exist in the mass range of $10^{1}-10^{2} M_{\odot}$ and supermassive black holes in the mass range of $10^{6}-10^{9.5} M_{\odot}$ [四]. There are some recent indications that black holes with mass in the range $10^{2}-10^{4} M_{\odot}$ may exist, but the evidence is not clear enough yet. Since black holes by definition have a deep potential well, it is only natural that black holes when they can be fed well should become power engines.

Accretion onto black holes is qualitatively different from that on other compact objects such as white dwarfs or neutron stars. White dwarfs and neutron stars have a well-defined surface at which all accreted material eventually should stop. This fixes the energy production efficiency $\varepsilon$ of the accretion. In addition, since the luminosity from the accretion should be in general less than the Eddington luminosity $L_{E} \equiv 4 \pi G M_{B H} m_{p} c / \sigma_{T h}$ (for pure hydrogen; $G$ is the Gravitational constant, $c$ speed of light, $M_{B H}$ mass of the accreting black hole, $m_{p}$ proton mass, $\sigma_{T h}$ Thomson cross section) for stable accretion, the mass accretion rate also should be less than the maximum mass accretion rate $\varepsilon^{-1} \dot{M}_{E}$ where the Eddington mass accretion rate $\dot{M}_{E} \equiv L_{E} / c^{2}$.

Black holes do not have a hard surface, and the efficiency is determined only by how much kinetic and thermal energy the accreted matter carries into the black hole horizon. The difference between the potential energy and the energy advected into the horizon is bound to be emitted during the accretion process. Theoretically, the efficiency $\varepsilon$ can be any value between 0 and 1 . The efficiency of 0 means matter carries all its rest mass energy into the horizon intact while that of 1 means all its rest mass energy has been emitted before entering the horizon. The black hole mass increases by the rest mass of accreted matter in the former case while no mass increase occurs in the latter case. The usual requirement that the luminosity should be less than the Edddington luminosity does not impose any a priori constraint on the mass accretion rate onto black holes unlike accretion onto white dwarfs or neutron stars because small enough efficiency can keep the luminosity below the Eddington limit even for a large mass accretion rate. Therefore, the accretion onto black holes can be much more diverse at least in terms of energy production efficiency and the mass accretion rate than that on white dwarfs or neutron stars.

The amount of advected energy is determined by the detailed physical, i.e., dynamical and thermal, state of the flow. The flow carries the kinetic and thermal energy of the flow inward in proportion to the radial velocity, which is determined by the radial pressure gradient as well as the rotation for a given gravitational field of the black hole. The amount of internal energy is affected by the radial velocity as well as the balance between heating and, generally radiative, cooling because the radial velocity determines the time of infall during which cooling or heating changes the internal energy of gas. Therefore, the accretion flow can assume many different states and properties: high or low mass accretion rate, high or low radiation efficiency, hot or cold, rotating or non-rotating, and spherical or disk-like.

When the microphysical processes within the accretion flow involve two particles only, such as particle-particle or particle-photon processes, the flow solution and its physical quantities become 
scale-independent, independent of the black hole mass, if the relevant quantities are scaled by the mass of the black hole. In such case, it is very useful to define dimensionless luminosity and mass accretion rate, $l \equiv L / L_{E}$ and $\dot{m} \equiv \dot{M} / \dot{M}_{E}$. However, free-free absorption is a three-body process and the flow is not scale-free if the flow is optically thick to the free-free absorption.

\section{Geometry of Accretion}

The accretion flow becomes spherical when the accreted matter has no angular momentum. Spherical geometry necessarily implies that any radiation produced at inner radii has to go through the accretion flow at outer radii. The interaction between radiation and matter may or may not play important role depending on the optical depth of the flow. The flow is mostly freely falling, and the radiation efficiency is small; matter does not have enough time to radiate. The flow itself, however, can be hot or cool. Bulk viscosity exists, but generally does not affect the dynamics.

When the accreting matter has angular momentum much larger than $r_{S c h} c$, where $r_{S c h} \equiv$ $2 G M / c^{2}$ is the Schwarzschild radius, the infalling matter should slow down in terms of radial motion well outside the horizon. If the accreting matter has no viscosity at all, the accretion stops at the centrifugal radius where the angular momentum becomes equal to the Keplerian angular momentum. If the accreting matter has viscosity, the angular momentum is transferred outward by the shear of the rotational motion and the accretion flow is spread out in radius and becomes disk-like. Since the rotational velocity of this accretion disk is close to the Keplerian rotation velocity, the radial motion becomes much smaller than the free-fall velocity, which provides enough time to radiate. The disk is cold and disk height is much smaller than the radius. The radiation efficiency of these 'thin' accretion disks can be quite high, up to 0.1 .

There certainly are intermediate cases where the rotational velocity of the accretion flow is not as high as the Keplerian rotation velocity but significant enough to affect the radial motion. This type of accretion flow assume roughly spherical or torus shape, yet often called disk.

\section{Spherical Accretion}

Spherical accretion flow with zero angular momentum is studied by Bondi [ [2] who elucidated important characteristics of spherical accretion. The relativistic version of spherical accretion was

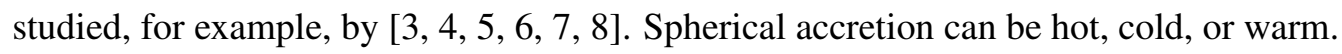

\subsection{Hot spherical accretion}

Spherical accretion flow remains hot when the dimensionless mass accretion rate $\dot{m} \ll 1$. The flow is almost freely falling and compressed adiabatically (Fig. 四). Cooling by bremsstrahlung and synchrotron may operate, but the flow does not have time to cool. The low density makes the radiation efficiency low $\varepsilon \lesssim 10^{-6}$. The adiabatic compression maintains the proton close to the virial temperature $T_{p} \sim T_{v i r} \equiv G M m_{p} /(2 k r)$ while the electron temperature follows that of the proton until it reaches and stays nearly constant at $T_{e} \sim 10^{10} \mathrm{~K}$ because multipole beyond dipole emission becomes very efficient near and above $T_{e} \sim m_{e} c^{2} / k$. These flow solutions are relevant to black holes accreting from interstellar or intergalactic medium. 


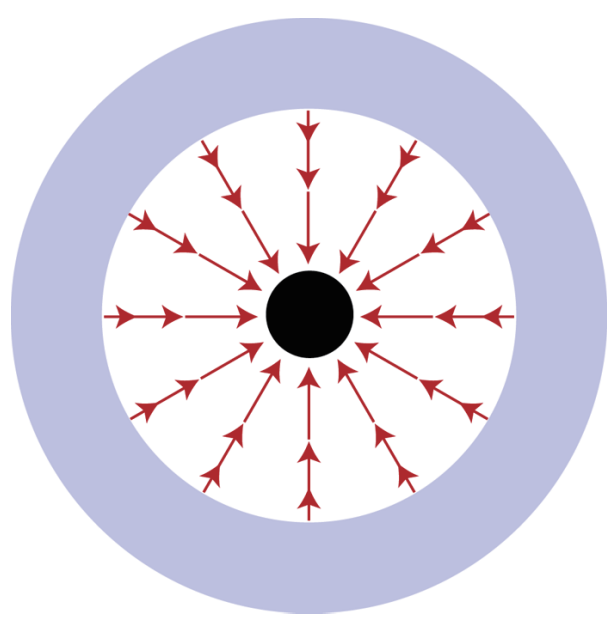

Figure 1: Spherical accretion onto a black hole surrounded by uniform gas.

\subsection{Cold spherical accretion}

As the mass accretion rate of spherical accretion becomes much larger than the Eddington accretion rate ( $\dot{m} \gg 1$ ), higher gas density makes radiative cooling efficient, and temperature is much less than the virial temperature, $T_{p}=T_{e} \sim 10^{4} \mathrm{~K}$ to $10^{6} \mathrm{~K}$. The flow becomes optically thick to electron scattering and free-free absorption. This optically thick accretion is called supercritical or hypercritical accretion. Radiation is in local thermodynamic equilibrium with matter and has

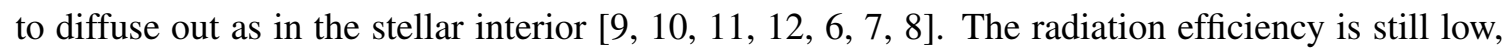
$\varepsilon \sim 10^{-6}$, due to high optical depth. Photon diffusion speed is roughly $c / \tau$ where $\tau$ is the optical depth. When $\tau$ becomes too large, the photon diffusion speed becomes smaller than the radial infall velocity, and photo trapping occurs [[13], [4]]. This regime requires fully relativistic radiative transfer treatment even though the velocity involved is much smaller than the speed of light [피, 미, ㅁ], \&, [16, [7]. If the mass accretion rate becomes extremely high, neutrino cooling can be important,

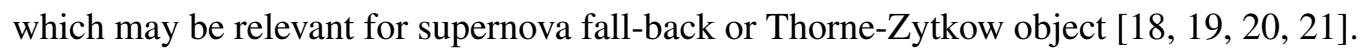

\subsection{Warm spherical accretion}

Atomic line cooling has a peak near $10^{4} \mathrm{~K}$, and accreting gas that is preheated by compression is either cooled to near $10^{4} \mathrm{~K}$ for cold spherical accretion or heated nearly to the virial temperature for hot spherical accretion. However, there exist an intermediate state where accreting flow is heated, for example through Compton scattering, by hot radiation produced at the inner region. This preheated warm accretion flow was first suggested by [22] and studied in detail by [ [6, [, , 8$]$. The radiation efficiency in this case is $\varepsilon \sim 10^{-4}$, much higher than the adiabatic hot accretion or cold accretion flow. This type of preheating is very important in understanding the feedback of a supermassive black hole onto its host galaxy [22]].

\section{Disk Accretion}

\subsection{Thin disk}

When the accreting gas maintains nearly Keplerian rotation, radial motion is minimal and 
generally ignored. The radial advection of gas entropy can be ignored and heating due to viscous dissipation is locally balanced by radiative cooling. Gas has enough density and time to cool and the gas temperature is low. Since the vertical structure of the disk is in hydrostatic equilibrium, low temperature means low vertical disk height and the disk is geometrically thin (Fig. \). Viscous stress transfers the angular momentum outward, which enables the gas to move inward. If viscous stress is proportional to the total pressure, the disk structure can be easily solved [24]. The radiation efficiency of the disk is determined by the location of the inner edge of the disk, and is generally quite high, $\varepsilon \sim 0.1$. This accretion flow can explain most galactic $\mathrm{X}$-ray sources.
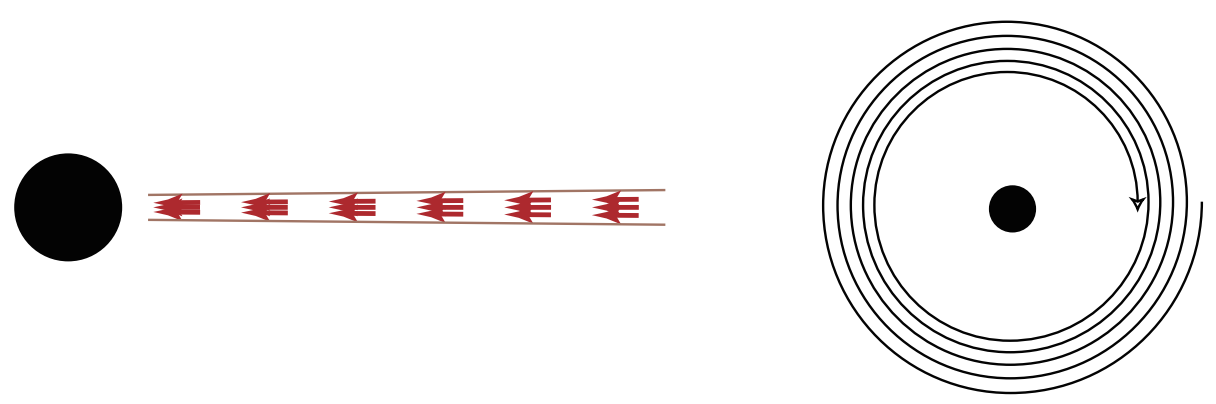

Figure 2: Thin disk seen from side (left) and from above (right).

\subsection{Thick disk}

As the mass accretion rate increase above $\dot{m}=\varepsilon^{-1}$, the temperature at the vertical center of the disk increases and radiation pressure becomes important as in massive stars. The vertical height of the disk then increases and the disk becomes thick and three-dimensional (Fig. [1]). The radial velocity becomes significant, reaching the sonic point within the flow while the rotation velocity is less than the Keplerian value [25, [26, 27]].

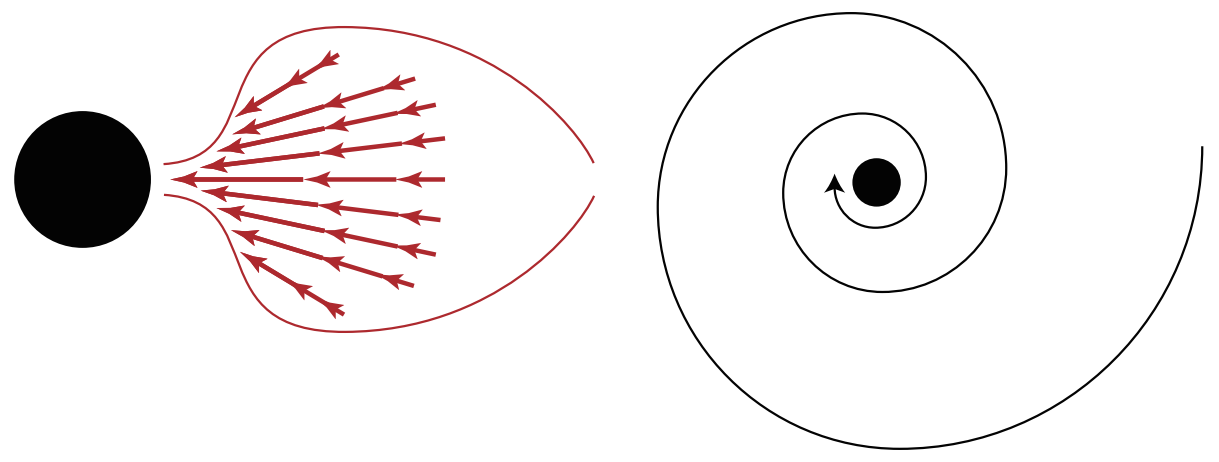

Figure 3: Thick disk seen from side (left) and from above (right).

\subsection{Slim disk}

Solving the three-dimensional thick accretion disk is not straightforward. Abramowicz et al. [28] used vertical height-integrated accretion disk equation and $\alpha$ viscosity description to study the super-Eddington $\left(\dot{m}>\varepsilon^{-1}\right)$ flow. Stable disk solutions with significant radial motion were 
found at high $\dot{m}$ regime where thin disk is known to be unstable. This family of disk solutions is called 'slim' disk because the disk is thick but slim enough to allow height-integrated simplification valid. The optically thick flow traps and advects the radiation and gas entropy into the hole while radiation leaks out through the surface of the disk. The luminosity increase stops slightly above the Eddington luminosity as the mass accretion rate $\dot{m}$ increases further, and the radiation efficiency $\varepsilon$ decreases as the mass accretion rate $\dot{m}$ increases. One thing to note from the slim disk study of [28] is the critical nature of the accretion flow with radial motion: the flow has to go through the critical point, generally at or close to the radius where the radial velocity becomes equal to the sound speed. Steady-state solution requires regularity at the critical point. This regularity condition functions as an additional boundary condition, and in the slim disk the angular momentum becomes an eigenvalue for given black hole mass, viscosity parameter $\alpha$, and mass accretion rate.
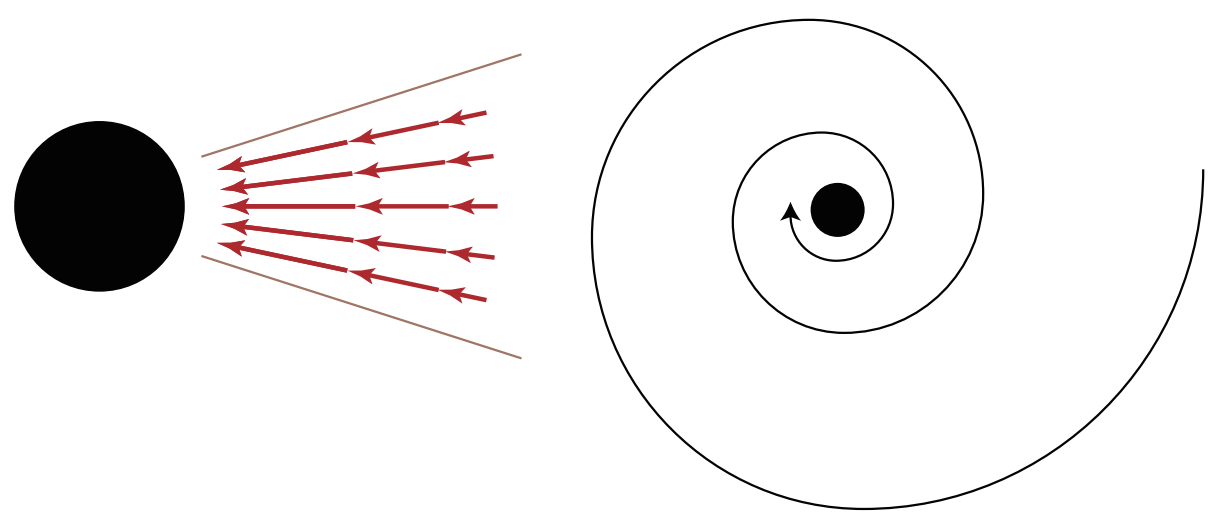

Figure 4: Slim disk seen from side (left) and from above (right).

\subsection{Radiatively inefficient accretion flow}

It took a few more years before people realized that the disk accretion flow can be like the spherical accretion flow in the sense that low density flow cannot cool efficiently and keep the gravitational binding energy in the form of gas thermal energy which will be transported inward with the flow, i.e. advected [ [29, BO, B]], just as in spherical accretion flow. In spherical accretion flow, it was natural to think that most of the gravitational binding energy is radially advected with the flow in the form of kinetic or thermal energy. In disk accretion flow, especially in the thin disk, viscous heating (originally from gravitational binding energy) is locally (in terms of radius) balanced by radiative cooling. However, significant radial motion makes both advection and radiative cooling relevant. In accretion disk terms, at a given radius viscous heating is balanced by radiative heating plus advective cooling. When the advective cooling is dominant, the disk becomes hot as in low $\dot{m}$ spherical flow, and called advection-dominated accretion disk (ADAF, Fig. (ل). Modified version of ADAF may be also possible such as convection-dominated accretion flow (CDAF [B2]). They are all low mass accretion flow with very high ion temperature. Yet radiative cooling is not efficient and the radiation efficiency is very low, therefore collectively called radiatively inefficient accretion flow (RIAF). The geometry of RIAF can be quasi-spherical or torus-like (Fig. (6)), as seen from the self-similar solutions of ADAF [BOB]]. 

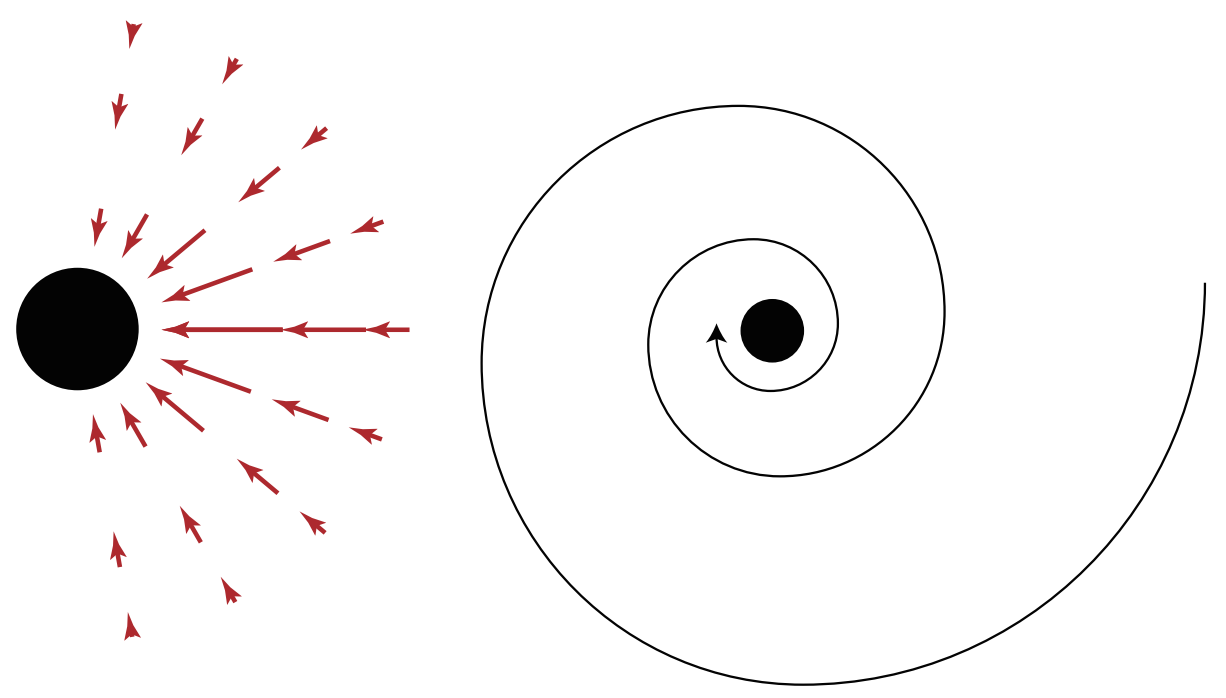

Figure 5: ADAF seen from side (left) and from above (right).
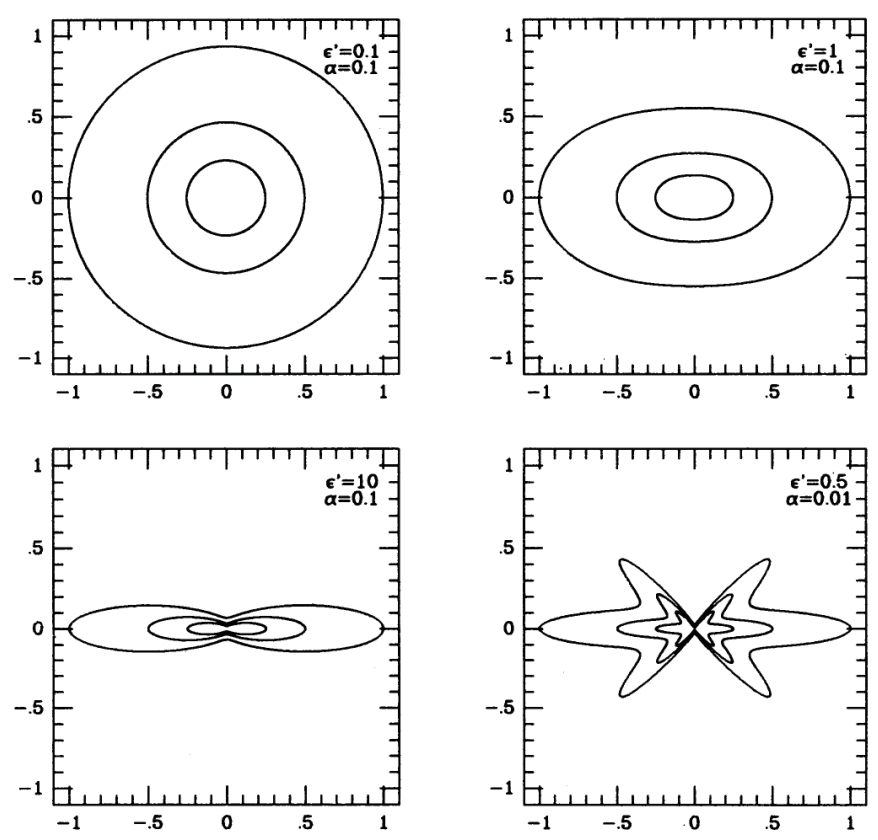

Figure 6: Isodensity contour of self-similar ADAF [RQ].

\subsection{Outflow}

RIAF is hot and, in addition, part of the binding energy of the inner part of the flow is transported to the outer parts through viscous torque. Therefore, gas is likely to become unbound [29, [3]]. Blandford \& Begelman [B3] showed that self-similar advection-dominated inflow-outflow solutions (ADIOS) are possible, in which outflow helps maintain the inward accretion. Recent numerial simulations also show that the net mass accretion rate can be as small as $\sim 0.01$ of the mass infall rate at the outer boundary [34]. 
RIAF is also a three-dimensional flow and any photons produced at smaller radii have to go through the outer part of the accretion flow as in the spherical accretion flow. Preheating of outer part of the flow can significantly change the physical properties of the flow. The flow also has a different density structure in poloidal direction. Therefore, the solution of RIAF has to include the effect of global heating in three-dimensional structure of the flow in addition to the advective cooling. Park \& Ostriker [35], 36, 37] have shown that the polar region of ADAF or CDAF can be radiatively heated beyond the virial temperature and the flow can develop outflow or wind.

\subsection{Luminosity and radiation efficiency}

Figure $\square$ shows aforementioned accretion flows in the mass accretion rate-luminosity ( $\log \dot{m}-$ $\log l$ ) plane [B]]. Diagonal lines are the constant radiation efficiency lines (solid for $\varepsilon=1.0$ and dotted for $\varepsilon=0.1$ ). Spherical accretion flows (crossses and trianlges [甘, $\mathbb{6}]$ ) generally have a luminosity well below the Eddington luminosity unless it is preheated (open circles [G, 四]) or is preheated and has abundant electron-positron pairs (small filled circles; [5]). The luminosity of the thin disk (dotted line), however, linearly increases with the mass accretion rate until the disk changes into a slim disk and the luminosity reaches the Eddington luminosity and saturates (dotdashed curve). ADAF (dashed line) starts near the spherical flow and approaches the thin disk as the mass accretion rate increases [ㅁ].

Figure 8 shows the flows in the radiation efficiency-luminosity $(\log \varepsilon-\log l)$ plane. Steadystate, hot or cold, spherical accretion flow has a radiation efficiency below $\varepsilon \sim 10^{-6.5}$. When preheated, it can reach up to $\varepsilon \sim 10^{-4}$. The radiation efficiency of ADAF increases with the luminosity, from $\varepsilon \lesssim 10^{-4}$ up to that of the thin disk, $\varepsilon \sim 0.1$. The radiation efficiency of the slim disk decreases from $\varepsilon \sim 0.1$ as the luminosity becomes larger than the Eddington luminosity.

\section{Mass Accretion Rate}

The mass accretion rate is the most important physical parameter in determining the luminosity and the radiation efficiency of the accretion flow, two most important parameters in understanding the feedback from and the growth of the black hole. Then the important question is what determines the mass accretion rate onto a black hole.

\subsection{Bondi Flow}

Bondi in his seminal work provided the answer to this question for the simplest case [వ]. $\mathrm{He}$ studied the polytropic gas (gas pressure is a power-law function of gas density only) accretion onto a Newtonian point mass, and found that four class of mathematical solutions exist: supersonic one in which gas is always supersonic, subsonic one in which gas is always subsonic, transonic one in which gas starts with subsonic speed at large radius, crosses the critical (sonic) point, and becomes supersonic at small radius, and non-physical one in which solutions exist only at a limited range in radius and multi-valued. He realized that the transonic solution has the maximum mass accretion for given boundary conditions, i.e., the density and temperature of the gas at infinity.

Since black holes do not have a hard surface to stop the flow, any accretion flow onto a black hole has to be transonic. Gas at infinity is at rest in pressure equilibrium state, but the gravitational pull from the black hole increases as the gas comes closer with slightly increasing density and 


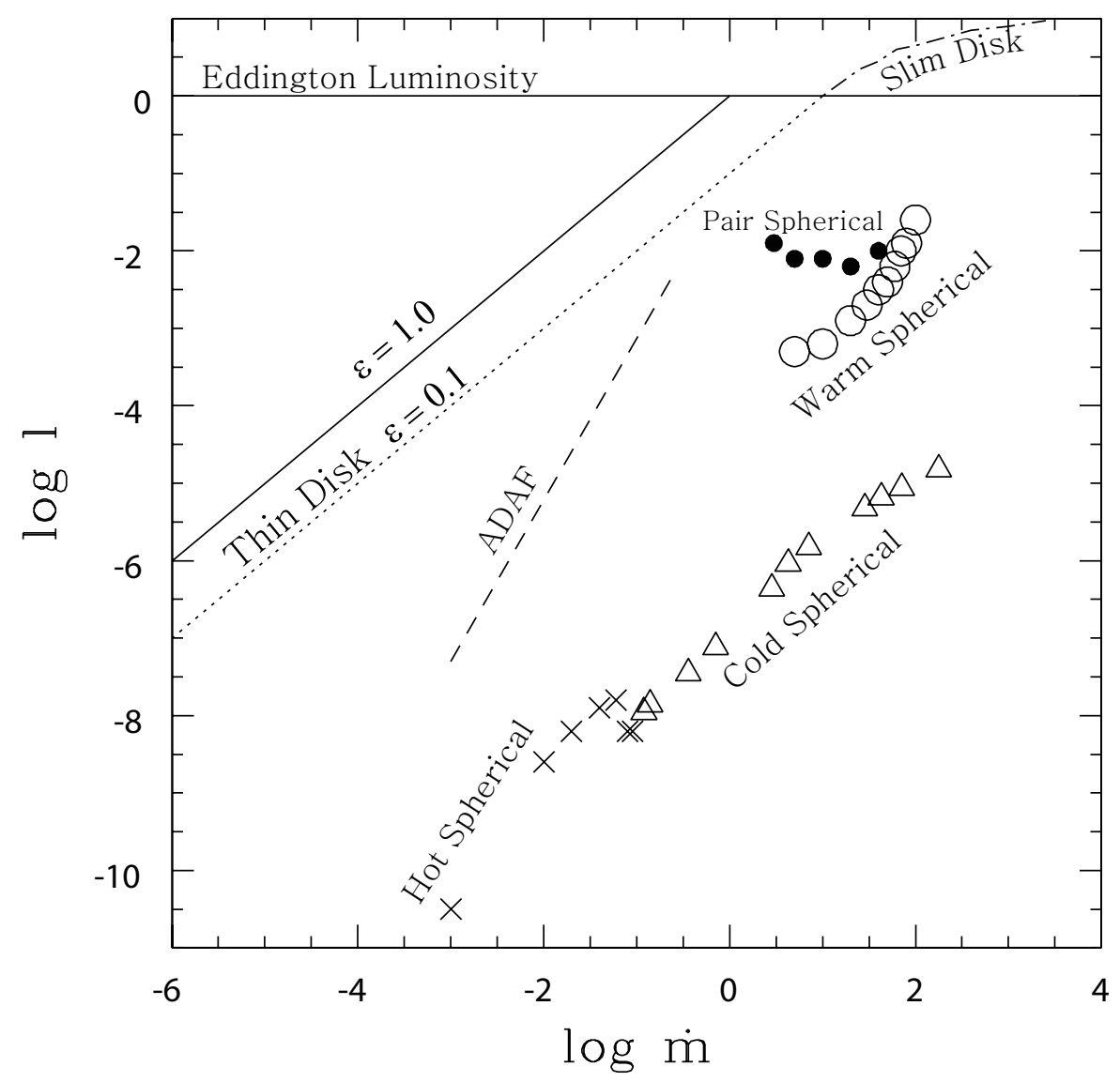

Figure 7: Various accretion flows in mass accretion rate $\left(\dot{m} \equiv \dot{M} / \dot{M}_{E}\right)$ versus luminosity $\left(l \equiv L / L_{E}\right)$ plane [B] $]$.

temperature to the Bondi radius $r_{B} \equiv G M_{B H} / c_{s, \infty}^{2}$, where $c_{s, \infty}$ is the isothermal sound speed of gas at infinity. Gravity starts to dominate inside $r_{B}$, and gas falls freely under the gravity of the black hole. The mass accretion rate in such case becomes so-called 'Bondi accretion rate'

$$
\dot{M}_{B}=\Lambda \gamma^{-3 / 2} 4 \pi r_{B}^{2} \rho_{\infty} c_{s, \infty}=4 \pi \Lambda \frac{(G M)^{2} \rho_{\infty}}{\gamma^{3 / 2} c_{s, \infty}^{3}}
$$

where $\rho_{\infty}$ is the mass density at infinity and $\Lambda$ is an order-of-unity function of the adiabatic index $\gamma$ [D]. The requirement that the flow be transonic is equivalent to the regularity condition at the sonic (critical) point, and it determines the mass accretion rate as an eigenvalue for a given density and temperature of gas at infinity. The value of $\gamma$ is $1 / 4$ for $\gamma=5 / 3$ and smoothly increases to 1.12 for the extreme value of $\gamma=1$. The higher the temperature of the gas is, the lower the Bondi rate is. Gravity affects smaller volume around a black hole for higher gas temperature. The numerical value of $\dot{M}_{B}$ is around $10^{-10} M_{\odot} \mathrm{yr}^{-1}$ for $10 M_{\odot}$ black hole accreting from $1 \mathrm{~cm}^{-3}, 10^{2} \mathrm{~K}$ gas. This accretion rate has been widely used, perhaps too widely used. Bondi's result assumes spherical accretion of gas with no angular momentum. However, accretion flow in real universe has some amount of angular momentum, in most cases greater than $r_{S c h} c$, and Bondi accretion rate may not be applicable at all. 


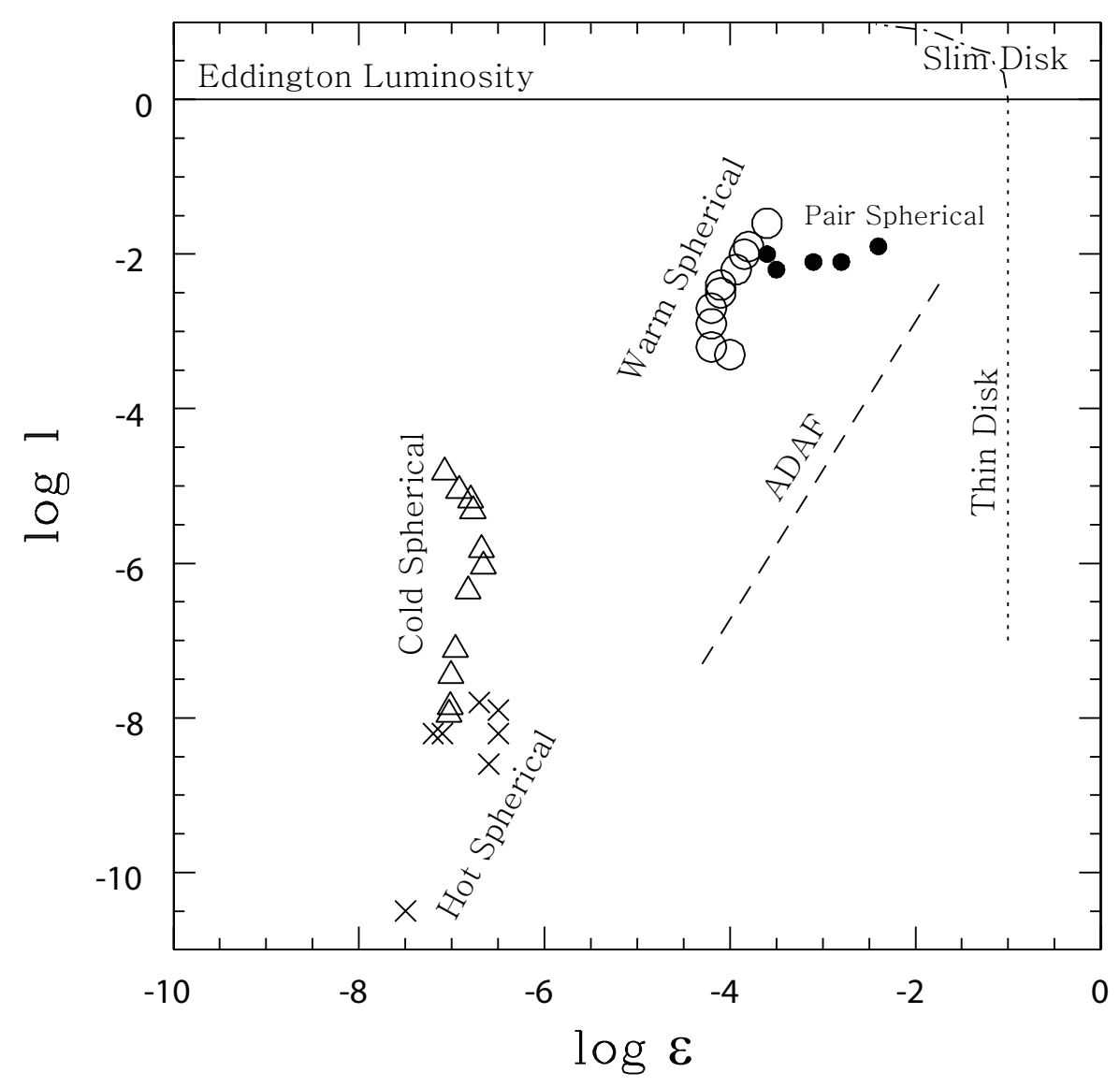

Figure 8: Various accretion flows in luminosity $(l)$ versus radiation efficiency $(\varepsilon)$ plane [B]].

\subsection{Generalized Bondi flow}

I [38] asked simple yet generally ignored question how the accretion flow behaves and what the mass accretion rate is if the accretion flow has angular momentum. This is a generalized version of Bondi accretion. Any rotating accretion flow has to lose angular momentum somehow. I used $\alpha$ viscosity prescription and slim disk approximation to find global, transonic, hot viscous accretion flow solutions. The radial momentum equation has a critical point, and regularity condition at the critical point requires that the solutions have the right mass accretion rate and the angular momentum for given gas density and temperature at infinity. In other words, not only gas density and temperature, as in Bondi flow, but also the gas angular momentum at the Bondi radius $r_{B}$ determines the mass accretion rate.

Figure $\theta$ shows the flow profiles of low angular momentum $(\lambda=0.14)$, intermediate angular momentum $(\lambda=0.27)$, and high angular momentum $(\lambda=1.7)$ flow, where $\lambda \equiv J_{\text {out }} / J_{B}$ is the ratio of the gas specific angular momentum at the outer boundary $r_{\text {out }}$ to the Bondi angular momentum $J_{B} \equiv r_{B} c_{S}\left(r_{B}\right)$ : Mach number in panel (a), radial velocity in units of $c$ in panel (b), angular momentum in units of $r_{S c h} c$ in panel (c) and temperature in panel (d). Low angular momentum flow is 
similar to the spherical accretion: the sonic point is located at a large radius and the Mach number is large and the angular momentum is much smaller than the Keplerian value at all radii. High angular momentum flow is similar to the ADAF with a sonic point very close the the Schwarzschild radius and angular momentum close to the Keplerian value at small radii.

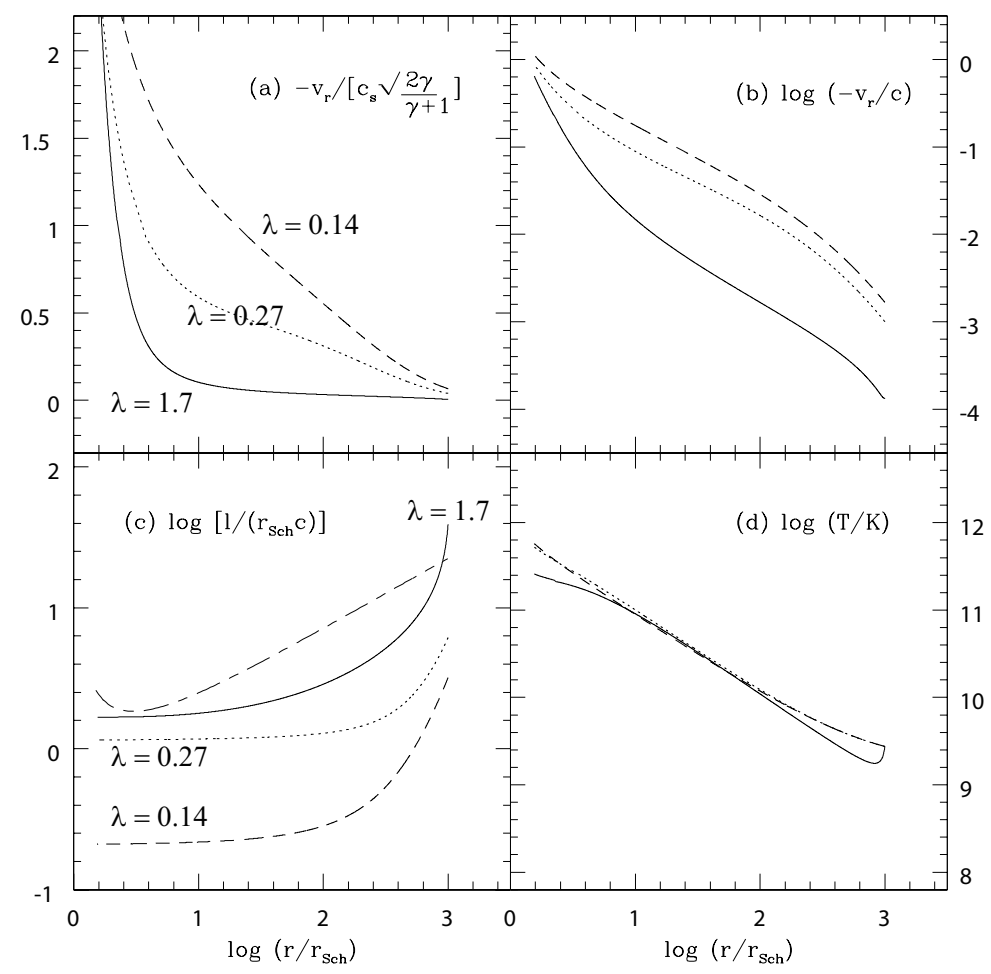

Figure 9: Flow profiles of low angular momentum $(\lambda=0.14)$, intermediate angular momentum $(\lambda=0.27)$, and high angular momentum $(\lambda=1.7)$ flow [B8]: Mach number in panel (a), radial velocity in unit of $c$ in panel (b), angular momentum in unit of $r_{S c h} c$ in panel (c) and temperature in panel (d).

One important realization from [B8] is that the mass accretion rate of the low angular momentum flow is equal to the Bondi accretion rate, but that of high angular momentum decreases as the angular momentum increases. The numerical solutions roughly obey the empirical formula

$$
\dot{M} / \dot{M}_{B}=0.09\left(\frac{\alpha}{0.01}\right)\left(\frac{J_{\text {out }}}{J_{B}}\right)^{-1} .
$$

Figure 10 shows the mass accretion rate $\dot{M} / \dot{M}_{B}$ as a function of angular momentum parameter $J_{\text {out }} / J_{B}$ for various values of $\alpha$ and gas temperature (different symbols). The value of $J_{\text {out }} / J_{B} \sim 1$ represents a flow close to the Keplerian rotation near the Bondi radius and $J_{\text {out }} / J_{B} \ll 1$ a flow with almost no rotation. If the accretion flow is rotating with the Keplerian speed $\left(J_{\text {out }}=J_{B}\right)$, the mass accretion rate can be only $9 \%$ of the Bondi accretion rate for $\alpha=0.01$. Higher value of viscosity parameter $\alpha$ decreases the discrepancy with the Bondi accretion rate because viscosity efficiently removes the rotational effect.

Although this work [38] adopted a simplified viscosity prescription and covered limited parameter space, it showed that the global solutions for hot, rotating viscous accretion flow exist from 


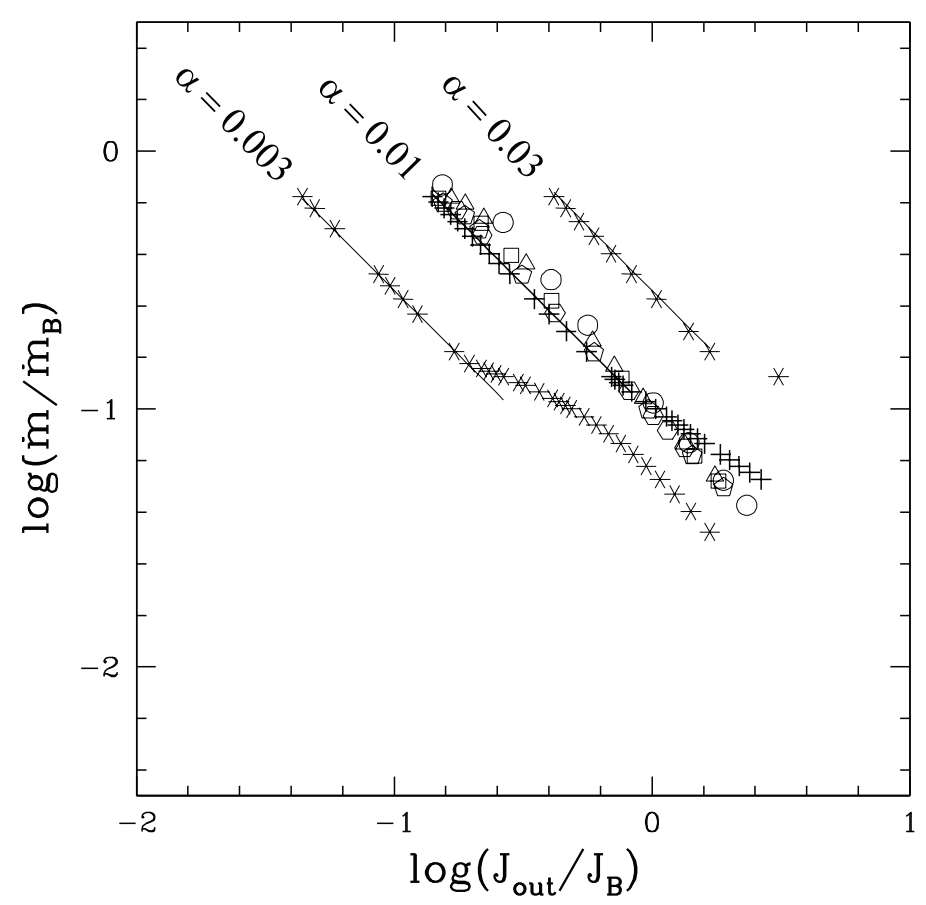

Figure 10: Mass accretion rate (in units of Bondi accretion rate) as a function of gas angular momentum (in units of Bondi angular momentum) [38]].

the Bondi flow to Keplerian disk continuously and its mass accretion rate depends on the angular momentum of the gas accreted and can be more than a factor of 10 smaller than the classic Bondi accretion rate.

Narayan \& Fabian [B9] subsequently applied similar approach to the cooling flow onto a supermassive black hole within an elliptical galaxy. They used a more refined viscosity prescription and numerical method, and confirmed most of the result in [38] is valid. They, however, pointed out that the decrease in the mass accretion rate as the gas angular momentum increases is not so significant as in [B8]: as the gas angular momentum increases by a factor of 100, the mass accretion rate decreases only by a factor of 4 (Fig $\mathbb{~} \mathbb{U}$, left panel). They suspected that the discrepancy might be due to the small value of $r_{B} / r_{S c h}$ in [B8], and raised a question what the mass accretion rate would be in a wider parameter space of $\alpha-J_{\text {out }} / J_{B}-r_{B} / r_{S c h}$. But their own result actually showed that the mass accretion rate can be much smaller than the Bondi accretion rate for small viscosity parameter $\alpha$ (Fig $\mathbb{~}$, right panel).

We recently carried out similar study on the viscous rotating accretion flow but covering a much larger parameter space [40]. We used slim disk formalism but with a polytropic equation of state to facilitate comparison with the original Bondi flow. Viscosity prescription of [38] is used. The outer boundary is set at the Bondi radius and Paczyński potential is used.

Figure $\square$ shows the mass accretion rate (in units of Bondi accretion rate) as a function of gas specific angular momentum for different values of $r_{B}$ and $\alpha$. The open circles are for $r_{B}=$ $2.5 \times 10^{3} r_{S c h}$ and filled circles for $r_{B}=5.0 \times 10^{4} r_{S c h}$. Circles connected by solid lines are for $\alpha=0.1$, those by dashed lines for $\alpha=0.03$, and those by dotted lines for $\alpha=0.01$. Despite 

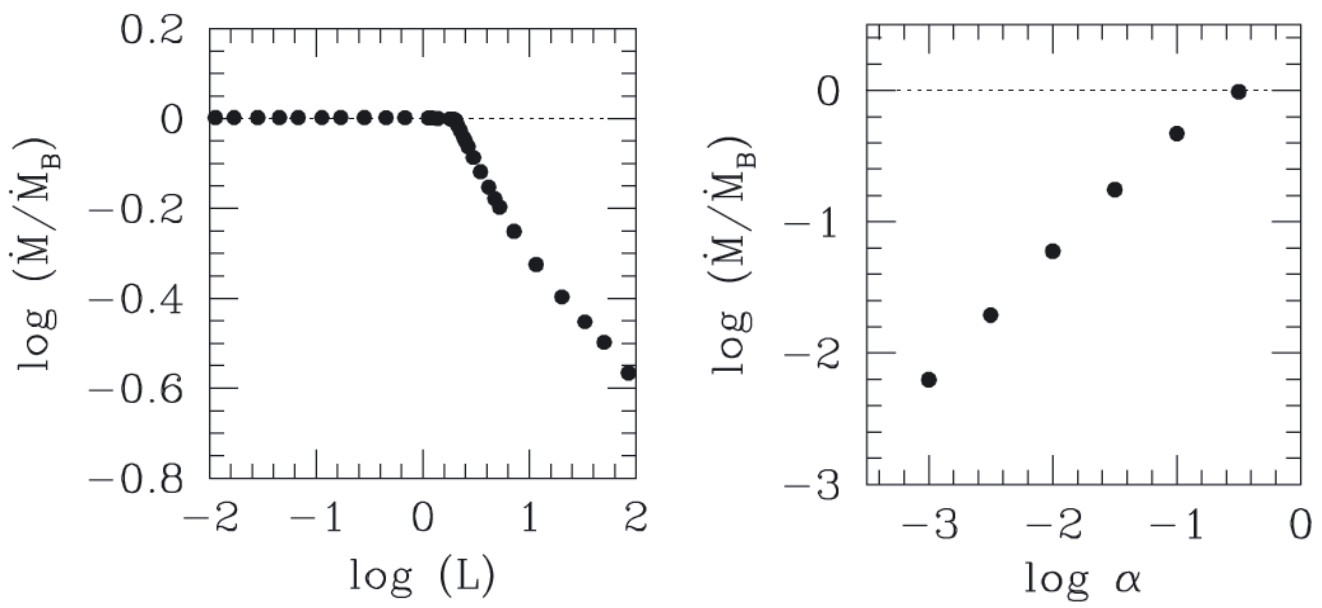

Figure 11: Mass accretion rate (in units of Bondi accretion rate) as a function of specific gas angular momentum (in units of $\sqrt{27 / 8} r_{S c h} c$ ) in the left panel and viscosity parameter $\alpha$ in the right panel [Bप].

various values of $\alpha$ and $r_{B}$, they all obey the empirical relation

$$
\dot{M} / \dot{M}_{B} \simeq 0.1\left(\frac{\alpha}{0.01}\right)\left(\frac{J_{\text {out }}}{J_{B}}\right)^{-1},
$$

(grey lines in Fig. [2), which is almost equal to the empirical relation found in [38].

Dependence of mass accretion rate on the adiabatic index is also studied. Figure [13] shows the mass accretion rate (in units of Bondi accretion rate) as a function of gas angular momentum (in units of the Bondi angular momentum) for different values of adabatic index $\gamma$ (solid line for $\gamma=1.553$, dashed for $\gamma=1.6$, and dotted for $\gamma=1.969$ ). The upper panels are for $\alpha=0.01$, lower for $\alpha=0.03$, left for $r_{B}=1.0 \times 10^{3} r_{S c h}$, and right for $r_{B}=2.5 \times 10^{3} r_{S c h}$. The mass accretion rate (in units of Bondi accretion rate) hardly changes for different value of the adiabatic index regardless of the viscosity parameter or the Bondi radius.

\subsection{Outflows}

By defnition, accretion flow implies flow moving radially inward, but outflow from hot RIAF seems normal rather than exception [20, [33]. So we implemented self-similar ADIOS of [B3]] into the slim disk equations and studied whether outflow changes the physical characteristics as well as the mass accretion rate [40]. For typical magnetic wind or gasdynamical wind, the flow profile does not change much. The flow is close to the ADAF, but with less angular momentum by the amount carried out by the wind. The mass accretion rate of ADIOS also follows the empirical relation $\dot{M} / \dot{M}_{B} \propto \alpha\left(\frac{J_{\text {out }}}{J_{B}}\right)^{-1}$ but with a larger coefficient because the angular momentum is effectively removed by the wind. Hence, the mass infall rate (at the Bondi radius) into the black hole increases when outflow develops. The final mass accretion rate across the black hole horizon, however, becomes only a small fraction of the original mass infall rate. The increase of mass infall rate at the Bondi radius is only a factor or few, but the final mass accretion rate across the inner boundary can be as small as $\left(r_{i n} / r_{B}\right)^{p}$ where $r_{i n}$ is the inner boundary of the flow which, in general, is a few times $r_{S c h}$. For $r_{B}=10^{3} r_{S c h}, r_{i n}=3 r_{S c h}$, and the mass accretion rate parameter $p=3 / 4$ [B3] , the factor 


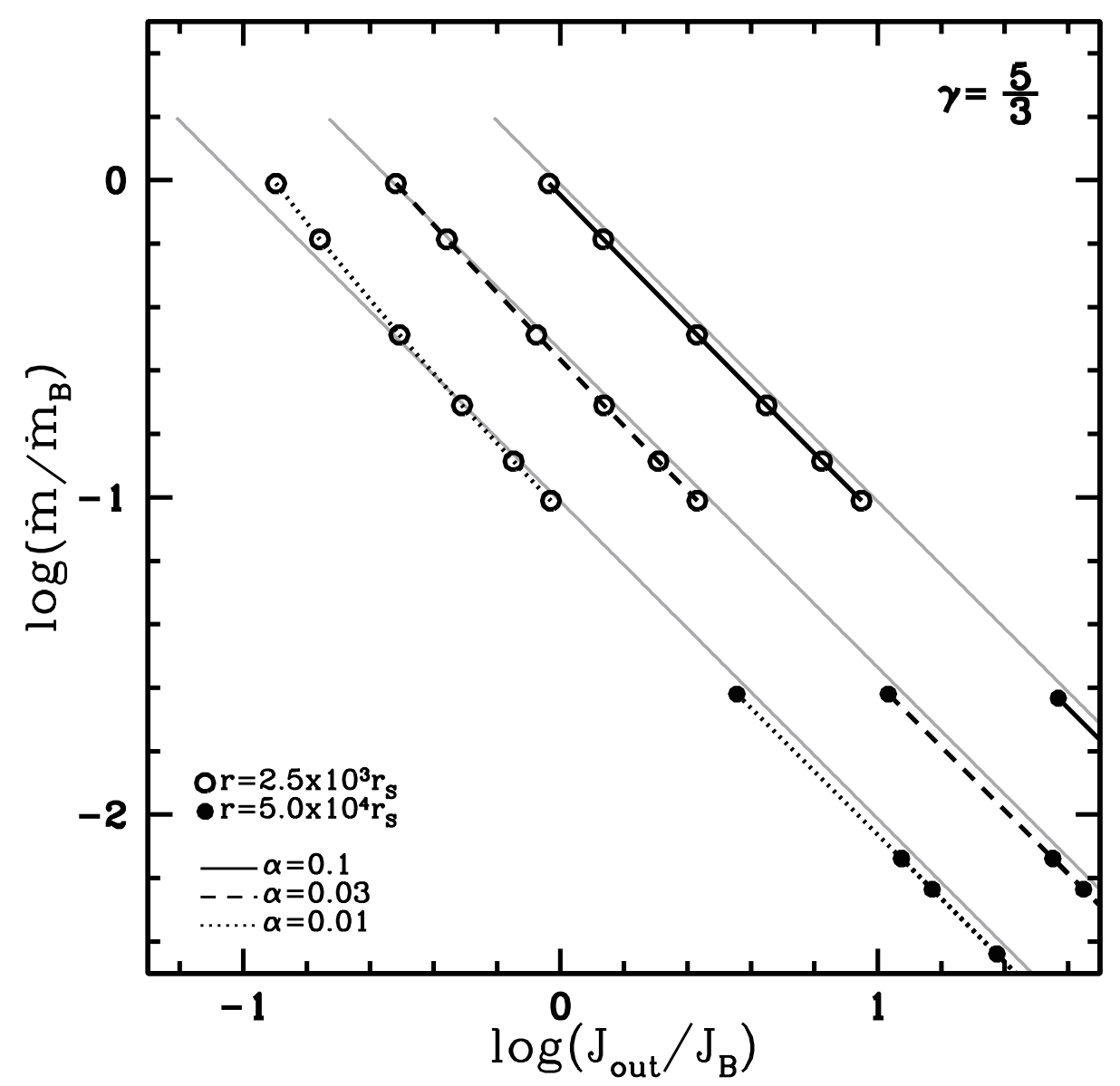

Figure 12: Mass accretion rate (in units of Bondi accretion rate) as a function of gas angular momentum (in units of the Bondi angular momentum) for different values of the Bondi radius and $\alpha$.

can be as small as 0.01 , meaning only a percent of mass infall at large radii actually crosses the horizon. The amount of radiation produced and the rate at which black hole grows will be severely limited.

\section{Summary}

Accretion onto black holes can take many different forms. It can have a widely different luminosity and energy production efficiency. It can be spherical or disk-like, hot or cold, optically thin or thick. Which type of flow can actually develop depends on the physical conditions of gas being accreted and physical processes involved. The mass accretion rate is also determined by the density, temperature, and angular momentum of gas. The mass accretion rate of rotating flow can be more than an order of magnitude smaller than the classic Bondi accretion rate. Outflow, ubiquitous feature of hot accretion flows, will further decrease the final mass accretion rate across the black hole horizon. The growth of supermassive black holes and their coevolution with the host galaxies can be fundamentally affected by the decreased mass accretion rate. 


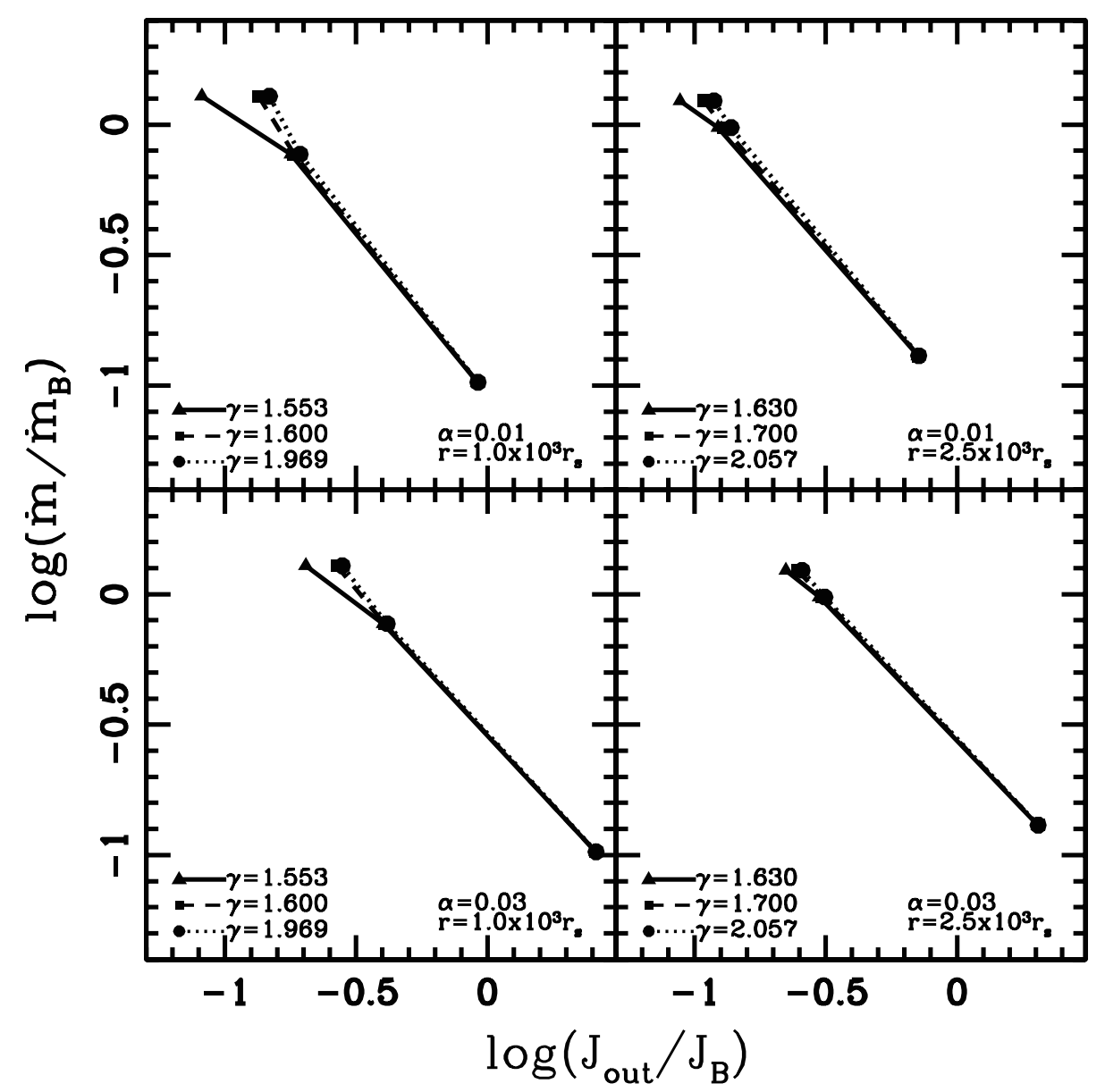

Figure 13: Mass accretion rate (in units of Bondi accretion rate) as a function of gas angular momentum (in units of the Bondi angular momentum) for different values of adiabatic index $\gamma$.

\section{Acknowledgments}

I thank the organizing committee of ICRC2017 for kind invitation and hospitality. This research was supported by Kyungpook National University Research Fund, 2016 and by the National Research Foundation of Korea to the Center for Galaxy Evolution Research (No.2017R1A5A1070354).

\section{References}

[1] R. Narayan and J. E. McClintock, Observational Evidence for Black Holes, in General Relativity and Gravitation: A Centennial Perspective Cambridge University Press [arXiv:1312.6698].

[2] H. Bondi, On Spherically Symmetrical Accretion, MNRAS 112 (1952) 195

[3] S. L. Shapiro, Accretion onto Black Holes: The Emergent Radiation Spectrum, ApJ 180 (1973) 531

[4] S. L. Shapiro, Accretion onto Black Holes: the Emergent Radiation Spectrum. II. Magnetic Effects, ApJ 185 (1973) 69

[5] M.-G. Park and J. P. Ostriker Spherical Accretion onto Black Holes - A New, Higher Efficiency Type of Solution with Significant Pair Production, ApJ 347 (1989) 679. 
[6] M.-G. Park, Self-consistent Models of Spherical Accretion onto Black Holes. I. One-temperature Solutions, ApJ 354 (1990) 64.

[7] M.-G. Park, Self-consistent Models of Spherical Accretion onto Black Holes. II. Two-temperature Solutions with Pairs, ApJ 354 (1990) 83.

[8] L. Nobili, R. Turolla, and L. Zampieri, Spherical Accretion onto Black Holes - A Complete Analysis of Stationary Solutions, ApJ 383 (1991) 250.

[9] M. H. Soffel, Stationary Spherical Accretion into Black Holes - The Transition from the Optically Thin to the Optically Thick Regime, $A \& A 116$ (1982) 111.

[10] R. A. Flammang, Stationary Spherical Accretion into Black Holes. II - Theory of Optically Thick Accretion, MNRAS, 199 (1982) 833.

[11] R. A. Flammang, Stationary Spherical Accretion into Black Holes. III Optically Thick Accretion in Particular Cases, MNRAS, 206 (1984) 589.

[12] J. M. Blondin, Hypercritical Spherical Accretion onto Compact Objects, ApJ, 308 (1986) 755.

[13] J. I. Katz, X-rays from spherical accretion onto degenerate dwarfs, ApJ, 215 (1977) 265

[14] M. C. Begelman, Black Holes in Radiation-dominated Gas - An Analogue of the Bondi Accretion Problem, MNRAS, 184 (1978) 53.

[15] K. S. Thorne, Relativistic radiative transfer - Moment formalisms, MNRAS, 194 (1981) 439.

[16] M.-G. Park, Relativistic Theory of Radiative Transfer: Time-dependent Radiation Moment Equations, A\&A, 274 (1993) 642.

[17] M.-G. Park, Equations of General Relativistic Radiation Hydrodynamics from a Tensor Formalism, MNRAS, 367 (2006) 1739.

[18] K. S. Thorne and A. N. Zytkow, Red giants and supergiants with degenerate neutron cores, ApJ, 199 (1975) L19

[19] R. A. Chevalier, Neutron star accretion in a supernova, ApJ, 346 (1989) 847.

[20] R. A. Chevalier, Neutron star accretion in a stellar envelope, ApJL, 411 (1993) L33.

[21] L. Zampieri, M. Colpi, S. L. Shapiro, I. Wasserman, Supernova Fallback and the Emergence of a Black Hole, ApJ, 505 (1998) 876.

[22] A. Wandel, A. Yahil, and M. Milgrom, Nonadiabatic Self-consistent Spherical Accretion as a Model for Quasars and Active Galactic Nuclei, ApJ, 282 (1984) 53.

[23] L. Ciotti and J. P. Ostriker, Radiative Feedback from Massive Black Holes in Elliptical Galaxies: AGN Flaring and Central Starburst Fueled by Recycled Gas, ApJ, 665 (2007) 1038.

[24] N. I. Shakura and R. A. Sunyaev, A Theory of the Instability of Disk Accretion on to Black Holes and the Variability of Binary X-Ray Sources, Galactic Nuclei and Quasars, MNRAS, 175 (1976) 613.

[25] B. Paczyński and P. Wiita, Thick accretion disks and supercritical luminosities, A\&A, 88 (1980) 23.

[26] M. Jaroszynski, M. A. Abramowicz, and B. Paczynski, Supercritical accretion disks around black holes, AcA, 30 (1980) 1.

[27] M. A. Abramowicz, M. Calvani, and L. Nobili, Thick accretion disks with super-Eddington luminosities, ApJ, 242 (1980) 772. 
[28] M. A. Abramowicz, B. Czerny, J. P. Lasota, and E. Szuszkiewicz, Slim Accretion Disks, ApJ, 332 (1988) 646.

[29] R. Narayan and I. Yi, Advection-dominated Accretion: A Self-similar Solution, ApJL, 428 (1994) L13.

[30] R. Narayan and I. Yi, Advection-dominated Accretion: Self-similarity and Bipolar Outflows, ApJ, 444 (1995) 231.

[31] R. Narayan and I. Yi, Advection-dominated Accretion: Underfed Black Holes and Neutron Stars, ApJ, 452 (1995) 710.

[32] R. Narayan, I. V. Igumenshchev, and M. A. Abramowicz, Self-similar Accretion Flows with Convection, ApJ, 539 (2000) 798.

[33] R. D. Blandford and M. C. Begelman, On the fate of gas accreting at a low rate on to a black hole, MNRAS, 303 (1999) L1.

[34] J. Li, J. Ostriker, R. Sunyaev Rotating Accretion Flows: From Infinity to the Black Hole, ApJ 767 (2013) 105.

[35] M.-G. Park and J. P. Ostriker Thermal Properties of Two-dimensional Advection-dominated Accretion Flow, ApJ 527 (1999) 247.

[36] M.-G. Park and J. P. Ostriker Preheated Advection-dominated Accretion Flow, ApJ 549 (2001) 100.

[37] M.-G. Park and J. P. Ostriker Compton-heated Outflow from Convection-dominated Accretion Flows, ApJ 655 (2007) 88.

[38] M.-G. Park, Mass Accretion Rate of Rotating Viscous Accretion Flow, ApJ 706 (2009) 637.

[39] R. Narayan and A. C. Fabian, Bondi flow from a slowly rotating hot atmosphere, MNRAS, 415 (2011) 372.

[40] M.-G. Park and D.-W. Han, Accretion onto Black Holes with Outflow, in proceedings of International Conference on Gravitation: Joint Conference of ICGAC-XIII and IK15. 